\title{
Evaluation of En-Face OCT Imaging in the Diagnosis of Macular Diseases
}

\author{
Yasser N. Elkholy ${ }^{\mathrm{a}}$, Ayman H. Nassar ${ }^{\mathrm{a}}$, Zeinab S. Elsanabary ${ }^{\mathrm{b}}$, Ahmed E. Daifalla ${ }^{\mathrm{a}}$, Elham \\ A. $\operatorname{Gad}^{\text {a }}$
}

a Department of Ophthalmology, Benha faculty of medicine, Banha University, Egypt.

${ }^{\mathrm{b}}$ Department of Ophthalmology, Faculty Of Medicine, Cairo University, Cairo, Egypt

Correspondence to: Yasser N. Elkholy, Department of Ophthalmology Benha faculty of medicine, Banha University, Egypt.

Email:

\section{Abstract}

Background: Optical coherence tomography (OCT) is a noninvasive scanning method that provides images with highresolution of the macula. B-scan OCT produces longitudinal cross-sectional images providing retinal layer analysis while en face OCT provides frontal surface and in depth images. Objectives: to assess the role of en Face OCT scanning in evaluation of some macular disorders like vitreo-macular interface and age related macular degeneration disorders. Methods: En Face OCT was used to scan the macular area of yasser_n_kholy@yahoo.com Received: 24 September 2021

Accepted: 11 December 2021 each patient, then the results were illustrated in the form of pathological signs that En face OCT can detect and confirmed by B-scan OCT and OCT angiography. Results: In this study 30 eyes of 24 patients; 14 females and 10 males were examined. Their age ranged from 26 to 83 years. Mean age was 64 years. 17 eyes with epi-retinal membranes and 13 eyes with AMD with en face OCT can detect multiple pathological signs. Conclusion: En face OCT can detect many useful signs if it is used solely, but it is much better to use it in combination with B-scan OCT.

Keywords: Structural enface OCT, enface OCT, vitreo-macular interface disorders, age related macular degeneration, $\mathrm{C}$-scan 


\section{Introduction}

Optical coherence tomography (OCT) is a noninvasive imaging modality presenting high-resolution scans of the macular area that has completely changed the field of ophthalmology (1).

B-scan OCT produces longitudinal crosssectional images helping in retinal layer micro-analysis. Improvement in data processing played an important role in en face OCT image formation, producing coronal scans of retinal and choroidal layers at the surface and multiple levels in depth. This offers additional benefits more than B-scan images as it gives an overview of multiple pathological structures in a single image (1).

We should distinguish between structural en face OCT which identifies the structure of the various retinal layers and en face OCT angiography which shows the retinal vessels and capillaries (2).

The epi-retinal membrane (ERM) pathology is a cellular proliferation that forms a semi-translucent, fibro-cellular proliferation on the inner retinal surface. ERMs have contractile cellular elements, that may cause retinal folding and increased macular thickness, thereby leading to affected visual acuity (VA), metamorphopsia, micropsia, and monocular diplopia. The majority of epiretinal membranes can be diagnosed by fundus examination on slit-lamp. They appear as a luster over the retina that causes retinal surface wrinkling. An ERM can be progressively more opaque, and so obscures retinal details and causes intraretinal edema (3).

Despite the widespread use of spectraldomain OCT in clinical practice, there is inadequate data on the incidence and visual effects of various vitreoretinal interface abnormalities. Much of the present epidemiological data are obtained from slit-lamp examination, either solely or combined with the grading of color fundus photographs, however many vitreoretinal interface abnormalities are not always identifiable using those methods (4).

Age-related macular degeneration (AMD) is a disease affected by multiple factors, that has an increasing prevalence all over the world and significant morbidity. The earliest studies carried out in the early 1980s, highlighted the significant hazard that AMD would represent in the following years. AMD is the chief reason of legal blindness in Western countries in people older than 50 years (5). 
En-face OCT scanning of the inner segment/outer segment (IS/OS) junction of retinal photoreceptors is a valuable tool to predict visual acuity and progression of geographic atrophy. En-face OCT offers high-resolution images that help in the diagnosis of different pathologies such as reticular pseudodrusen (RPD) and abnormal neovascularization of the choroid, which may be used as valuable markers for disease monitoring (1).

This study aimed at assessing the value of en Face OCT scanning in evaluation of vitreo-retinal interface disorders and age related macular degeneration disorders, both the wet and the dry types.

\section{Patient and methods}

The study involved 30 eyes of 24 patients with macular disorders involving the vitreo-retinal interface and age related macular degeneration.

\section{Design}

A prospective, cross sectional study.

\section{Inclusion criteria}

Patients above 18 years old diagnosed to have vitreoretinal interface disorders and AMD on clinical examination by slit lamp bio-microscopy and fundus examination.

\section{Exclusion criteria}

Patients with dense media opacity that interfere with imaging and uncooperative patients.

This study was conducted between Department of Ophthalmology, Faculty of Medicine, Benha University and Bostan diagnostic eye center in Cairo during the period from July 2019 to September 2020. Cases was collected and examined at Bostan diagnostic eye center, scans and results was examined and revised with investigators at ophthalmology department Benha faculty of medicine. The imaging technique was examined to the patients. This study was approved by the institutional ethical committee.

\section{Methods}

All patients were subjected to the following:

a) Detailed history taking including:

- Demographic data including age and gender

- Visual complaints

- Previous eye disease or surgery

b) Slit lamp bio-microscopy and fundus examination, to assess the anterior and posterior segment abnormalities. 
c) OCT examination using en face scans for the macular area performed using Optovue instrument Avanti ${ }^{\circledR}$ and confirmed by B-scan OCT and OCT angiography.

Eyes were dilated before en face OCT examination. All scans centered on the fovea by providing a central internal fixation mark.

This study was conducted during the period from July 2019 to September 2020.

\section{Main Outcome Measures:}

En face OCT photos were examined and different pathological findings were detected and results were recorded helped by fundus examination, B-scan OCT and OCTA.

Results were interpreted as the ability of en face OCT to catch different pathological findings.

\section{Results}

In this study, 30 eyes of 24 patients; 14 females and 10 males were examined. Their age ranged from 26 to 83 years with a mean age of 64 years.

Visual acuity also varied according to the pathology, ranging from 0.8 decimal to HM.
Different signs of each entity were detected as follows:

1. Epi-retinal membrane (ERM) (17 eyes).

2. Age-related macular degenerations (13 eyes) :

i. Active choroidal neo-vascular membrane (Active CNVM) (1 eye)

ii. Geographic atrophy (GA) (2 eyes)

iii. Drusen and drusenoid PED (9 eyes)

iv. Fibro-vascular pigment epithelium detachment (PED) (1 eye)

Results of eyes with different signs of ERM are explained in table 1 and those for cases of age-related macular degeneration are explained in table 2.

Table 1: showing different signs of epi-retinal membrane as detected by en face OCT.

\begin{tabular}{|l|l|}
\hline \multicolumn{2}{|l|}{ Epi-retinal membrane cases } \\
\hline Total & 17 \\
\hline Star fold & 2 \\
\hline plaque & 3 \\
\hline Diffuse folding & 10 \\
\hline Mixed & 1 \\
\hline Retinal window & 2 \\
\hline Not detected & 1 \\
\hline Affected fovea & 12 \\
\hline Fovea not affected & 5 \\
\hline
\end{tabular}


Table 2: showing different signs of age related macular degeneration as detected by en face OCT.

\begin{tabular}{|l|l|}
\hline \multicolumn{2}{|l|}{ Age related macular degeneration cases } \\
\hline Total & 13 \\
\hline Small drusen & 10 \\
\hline Large drusen & 7 \\
\hline Drusenoid PED & 6 \\
\hline Fibro-vascular PED & 1 \\
\hline Active CNVM & 1 \\
\hline Geographic atrophy & 2 \\
\hline
\end{tabular}

\section{Sample cases}

1. A plaque pattern of ERM with radiating folds

Figure 1: Right eye of a diabetic male patient 32 years old. Enface OCT image at level of ILM showing an
Sample cases showing different signs are showed as following:

1. A plaque pattern of ERM with radiating folds (figure 1)

2. Diffuse folding pattern of ERM with cystoid edema (figure 2)

3. Star fold pattern of ERM (figure 3)

4. Diffuse folding pattern of ERM with retinal window (figure 4)

5. Geographic atrophy (figure 5)

6. Drusen with different sizes and drusenoid PEDs (figure 6)
7. Active CNVM (figure 7) an Quality 6/10

Sco En Face $\mid$ Thickness $\mid$ RPE Elevation $/$ Color Overlay

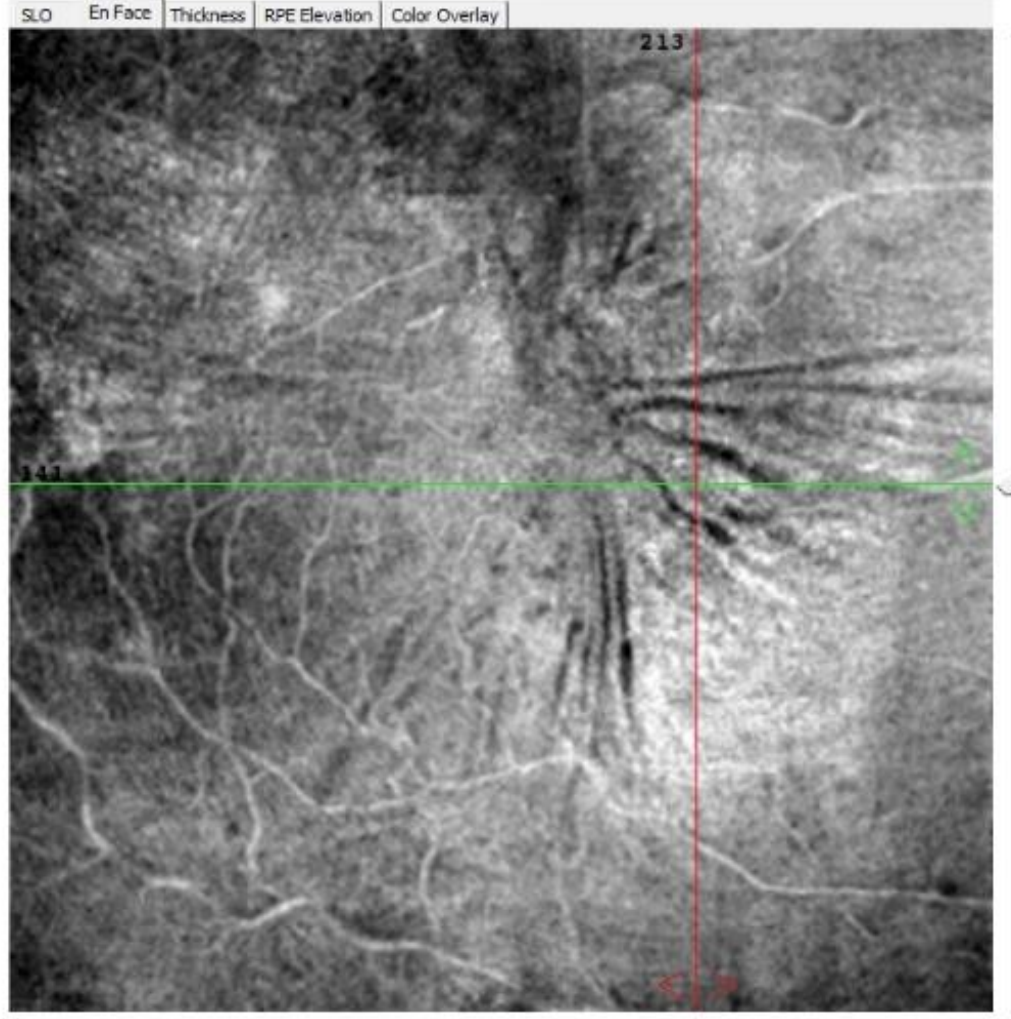

ERM plaque with radiating folds showing the extent and location of ERM.

\section{Right / OD}

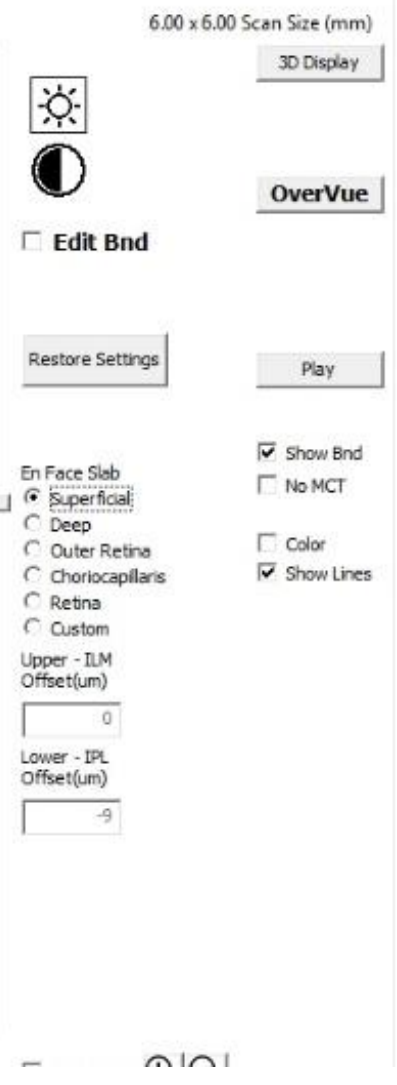



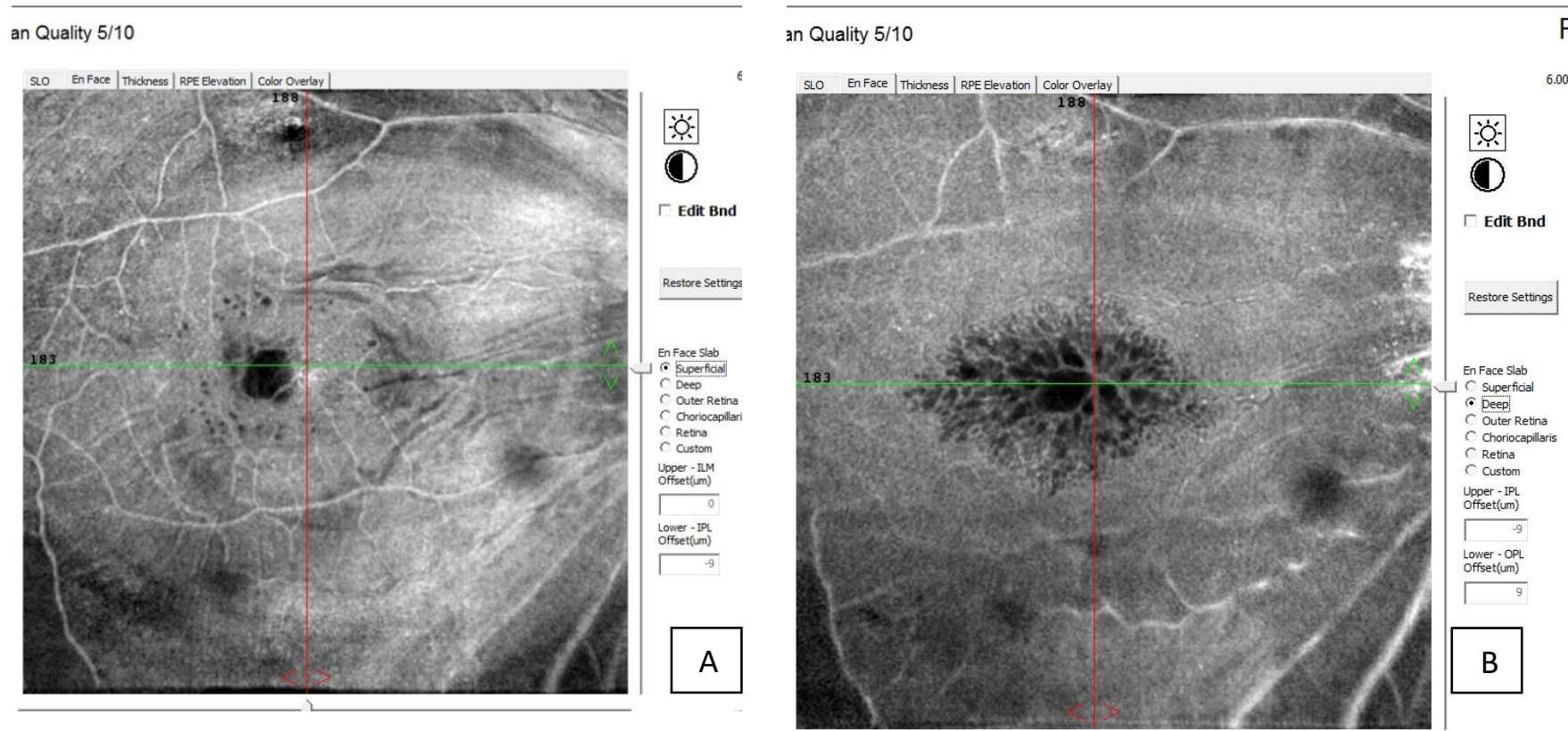

2. Diffuse folding pattern of ERM with cystoid edema

Figure 2: Right eye of a male patient 63 years old. En Face OCT image at level of superficial capillary plexus showing diffuse folding pattern with small cystic spaces(A), and at level of deep capillary plexus showing cavities of cystoid macular edema (B).

:an Quality 7/10

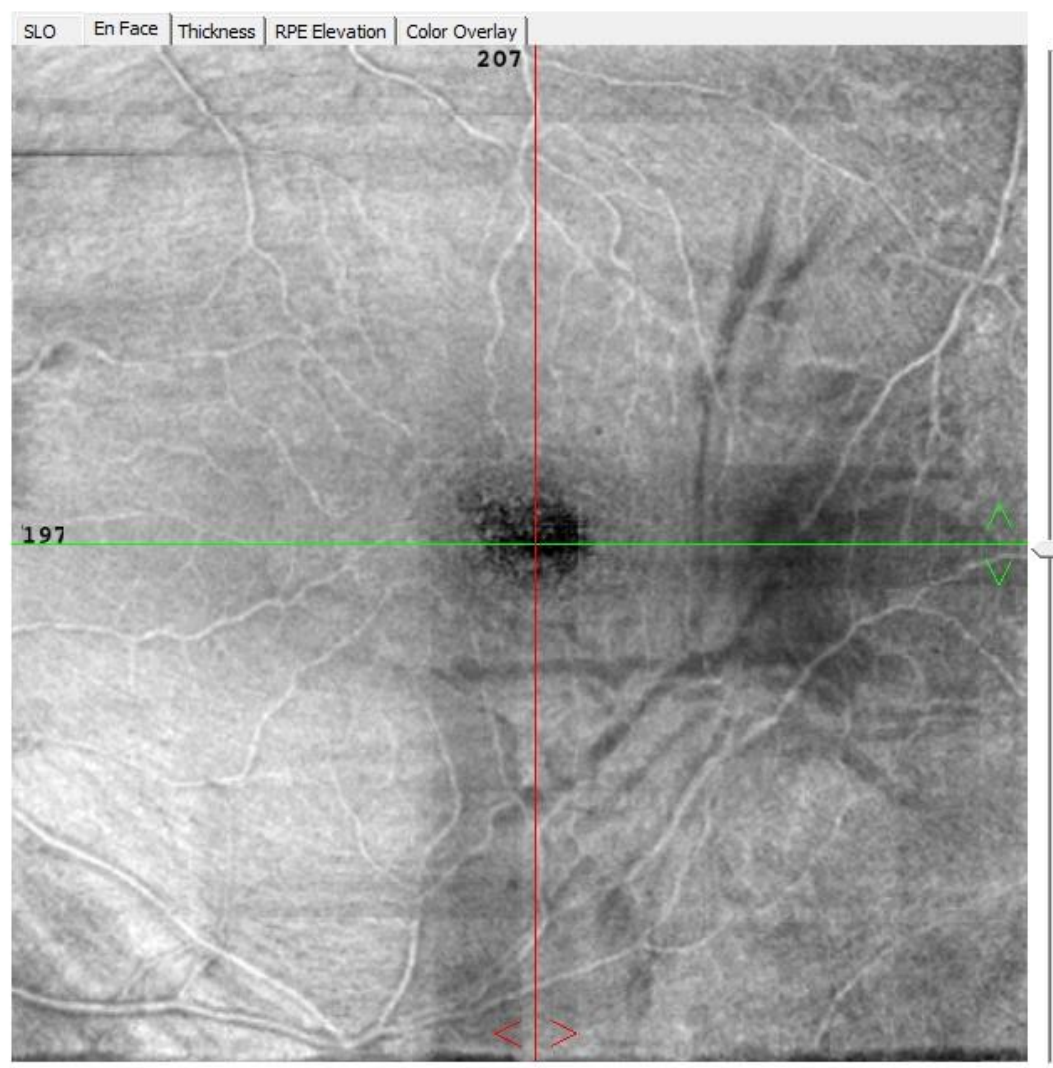

\section{Left / OS}

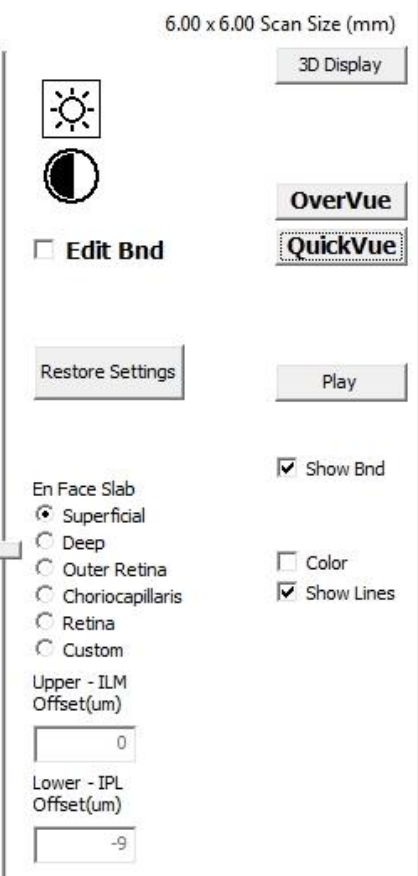

\section{Star fold pattern of ERM}

Figure 3: left eye of a male patient 75 years old. En Face OCT image at level of superficial capillary plexus showing a star fold pattern 
4. Diffuse folding pattern of ERM with retinal window

Figure 4: left eye of a male patient 61 years old. Enface OCT image at level of ILM showing a diffuse folding pattern with retinal window (yellow arrow) detected supero-nasal to the fovea (red arrow) showing the extent and location of ERM

:an Quality 6/10

Left / OS
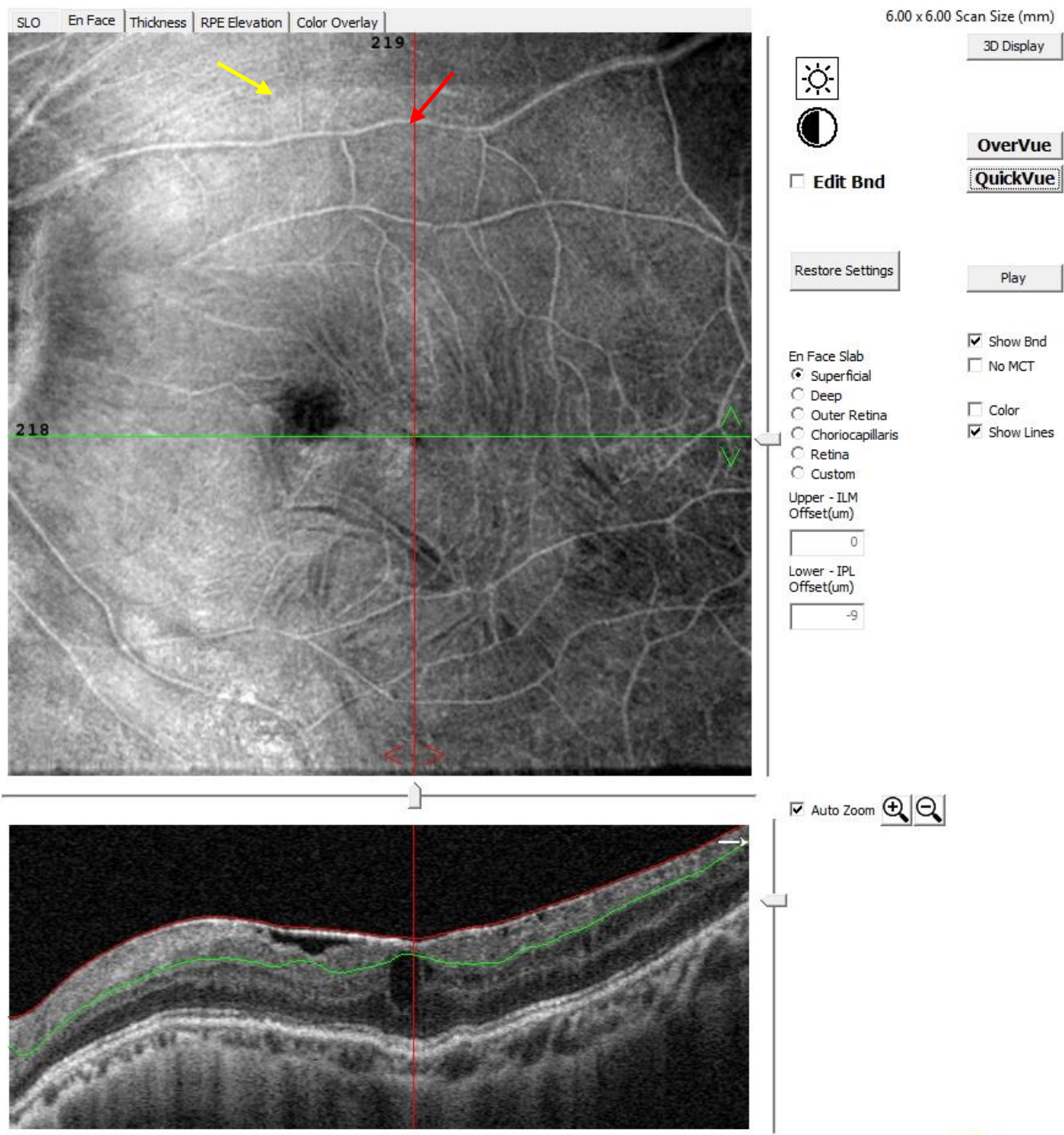

V Auto Zoom $\oplus \mid \Theta$

C Retina

Upper - ILM

Offset(um)

Lower - IPL

Offset(um)

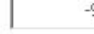

Comment 


\section{Geographic atrophy}

Figure 5: Left eye of male patient 83 years with geographic atrophy. enface OCT scan at level of choriocapillaris showing patches of loss of RPE and chorio-capillaris with visualization of large choroidal vessels denoting geographic atrophy.

an Quality $9 / 10$

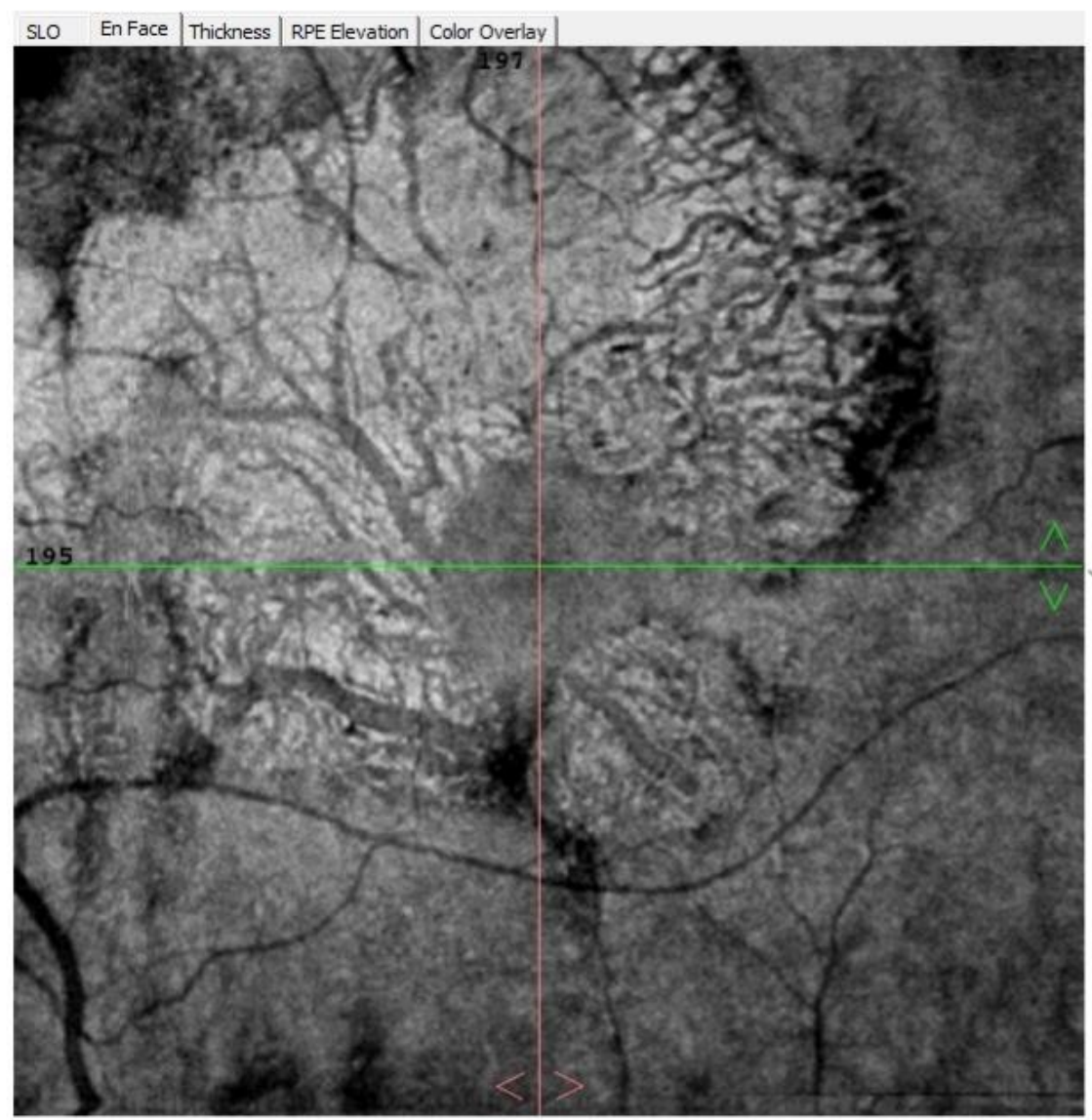

Left / OS

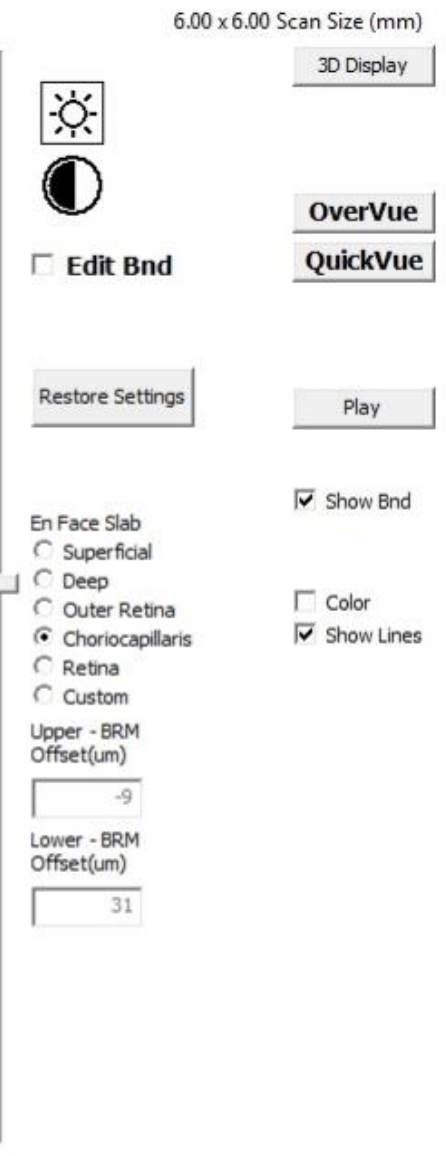


5. Drusen with different sizes and drusenoid PEDS

Figure 6: Right eye of a female patient 52 years old. Enface OCT scan at level of outer retina (A) showing sub and para-foveal different sized whitish spots of drusen and drusenoid PEDs and at level of chorio-capillaris $(B)$ showing back shadowing of effect
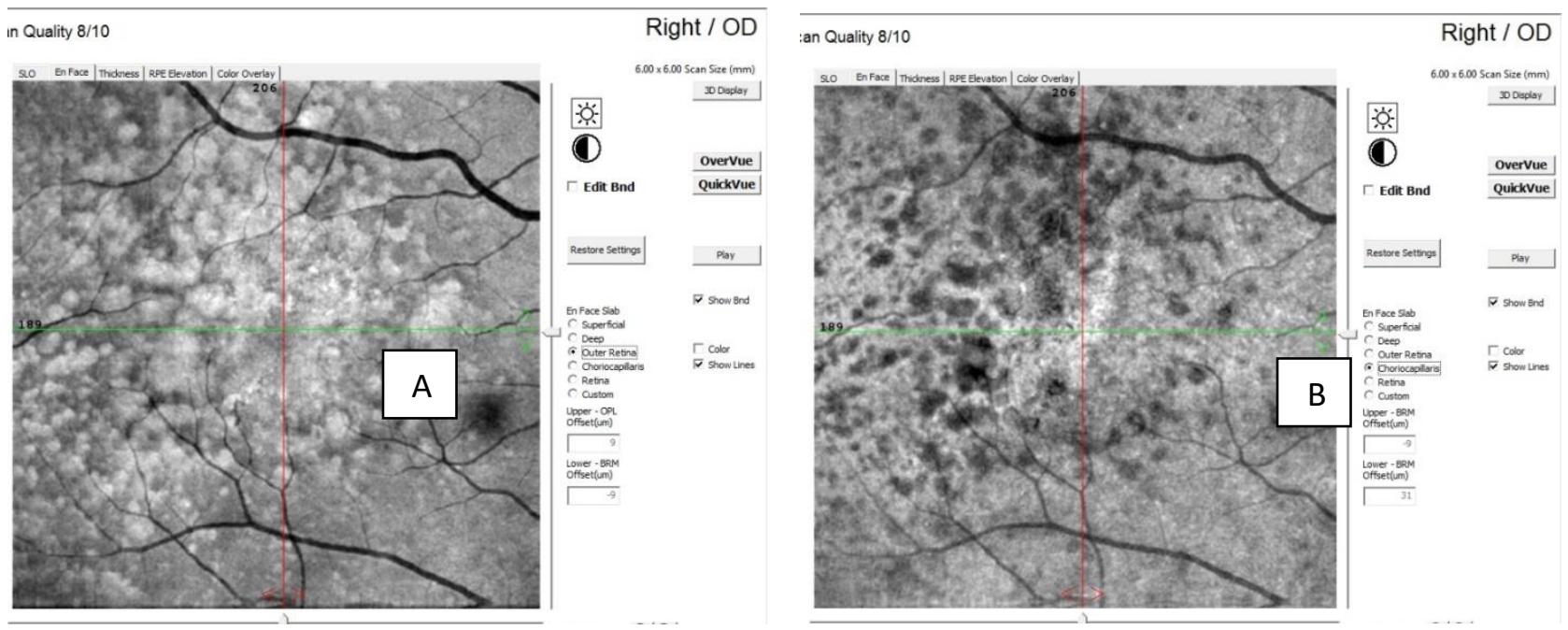


\section{Active CNVM}

Figure 7: right eye of male patient 65 years old. En face OCT image at the outer retina level showing CNVM with adjacent neuro-sensory detachment denoting activity (proved by OCTA).

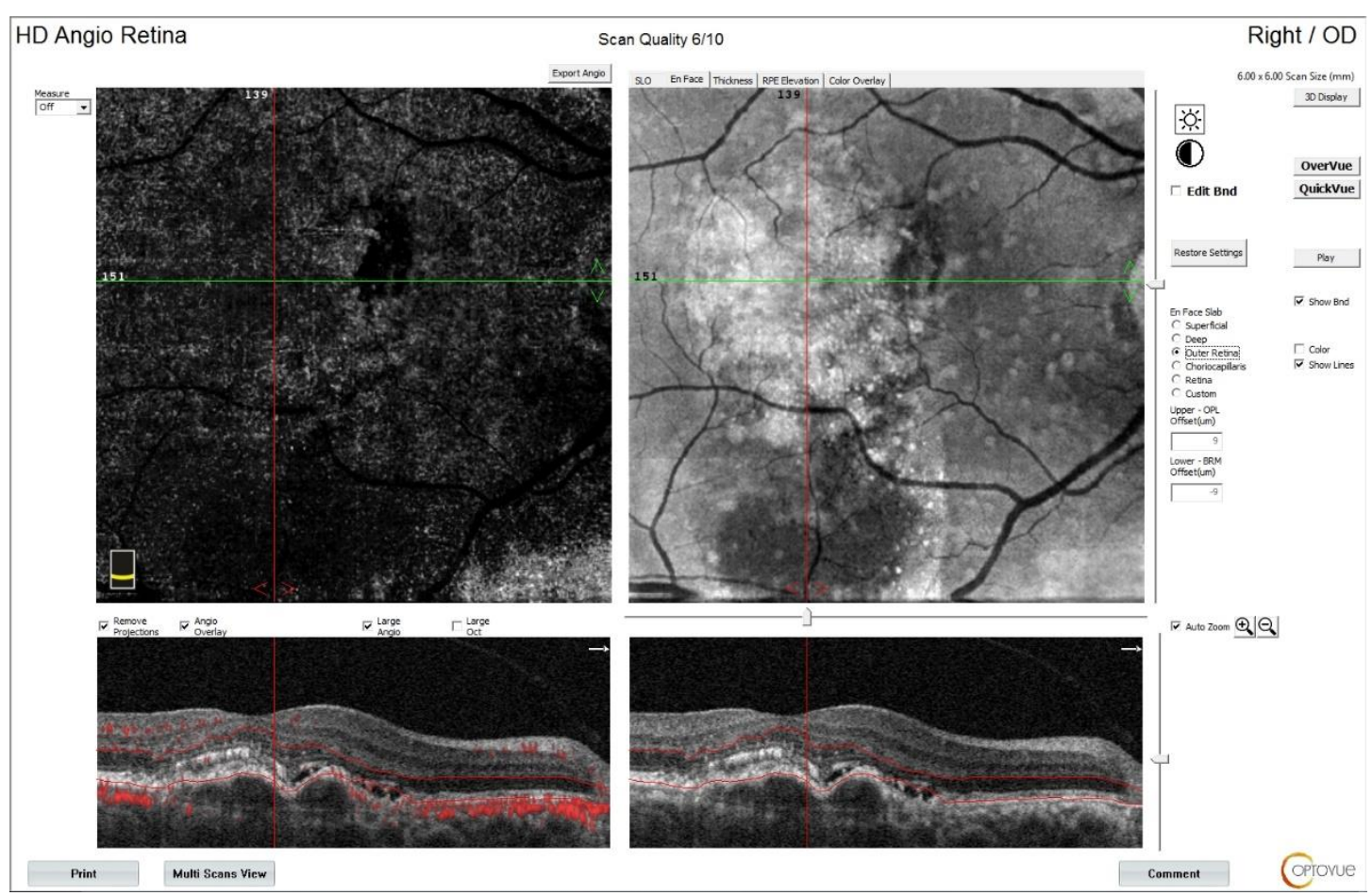


an Quality 6/10

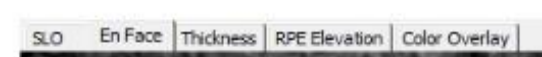

\begin{tabular}{cc|c|c|c|} 
Sco En Face & Thickness & RPE Elevation & Color Overlay
\end{tabular}

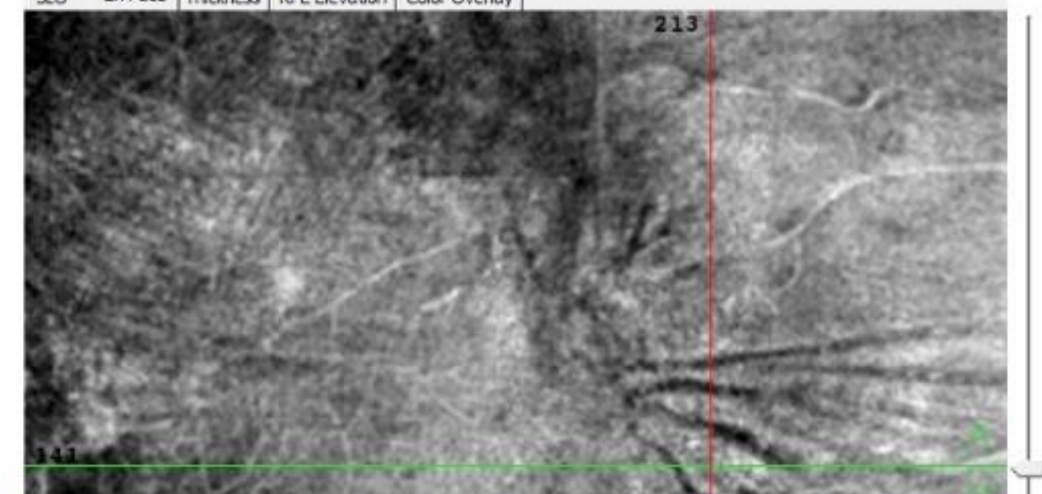

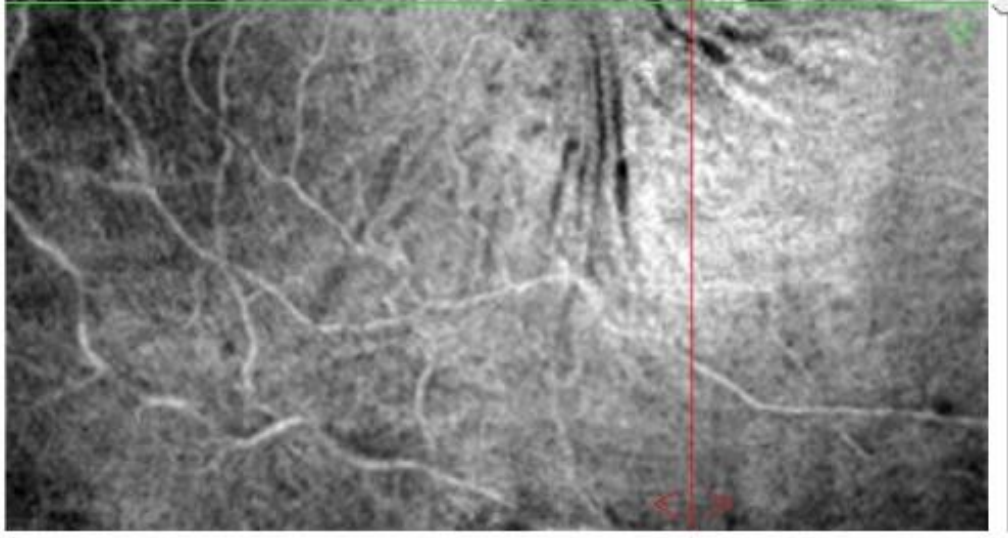
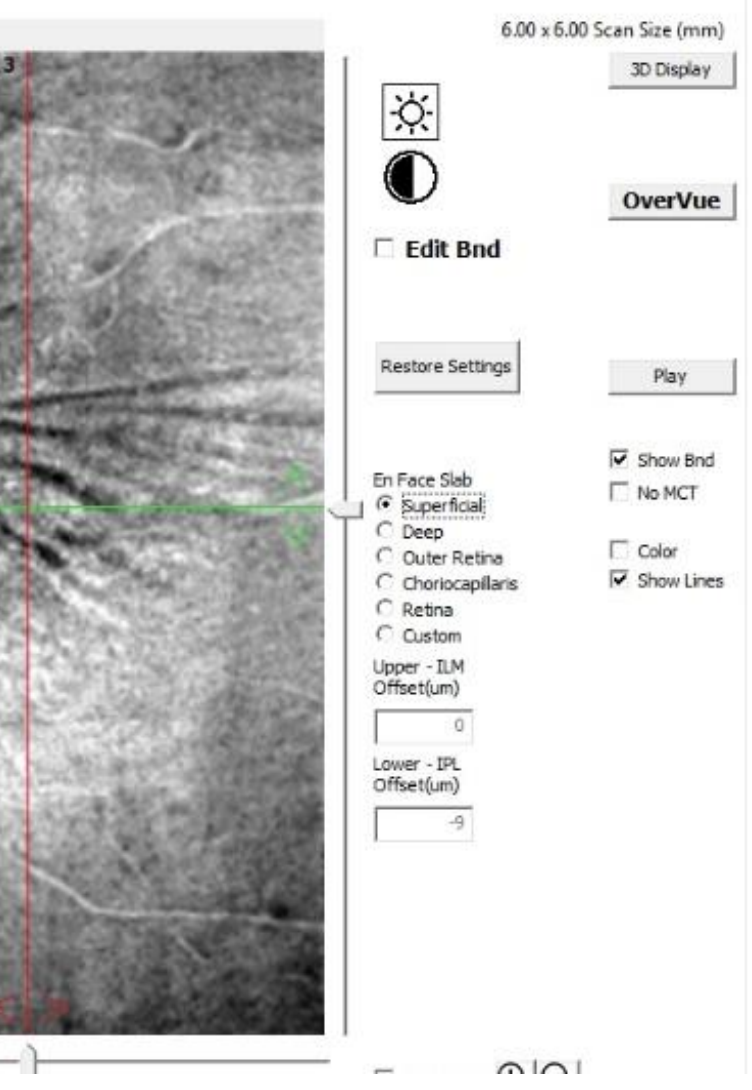

7. A plaque pattern of ERM with

8. radiating folds

Figure 1: Right eye of a diabetic male patient 32 years old. Enface OCT image at level of ILM showing an ERM plaque with radiating folds showing

the

extent

and 
9. Diffuse folding pattern of ERM with cystoid edema
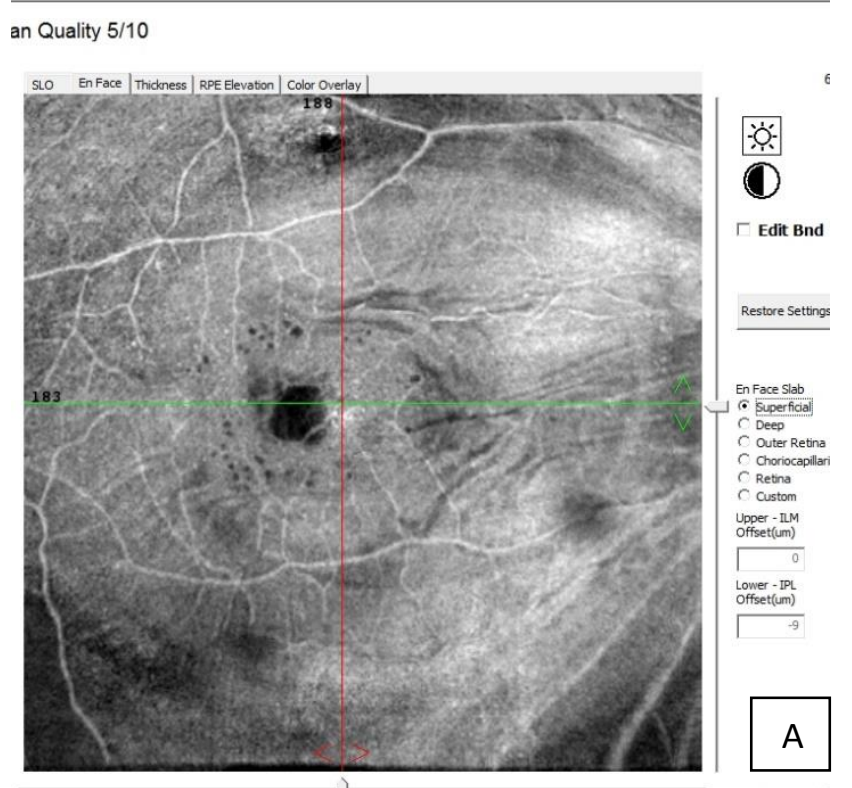
an Quality $5 / 10$

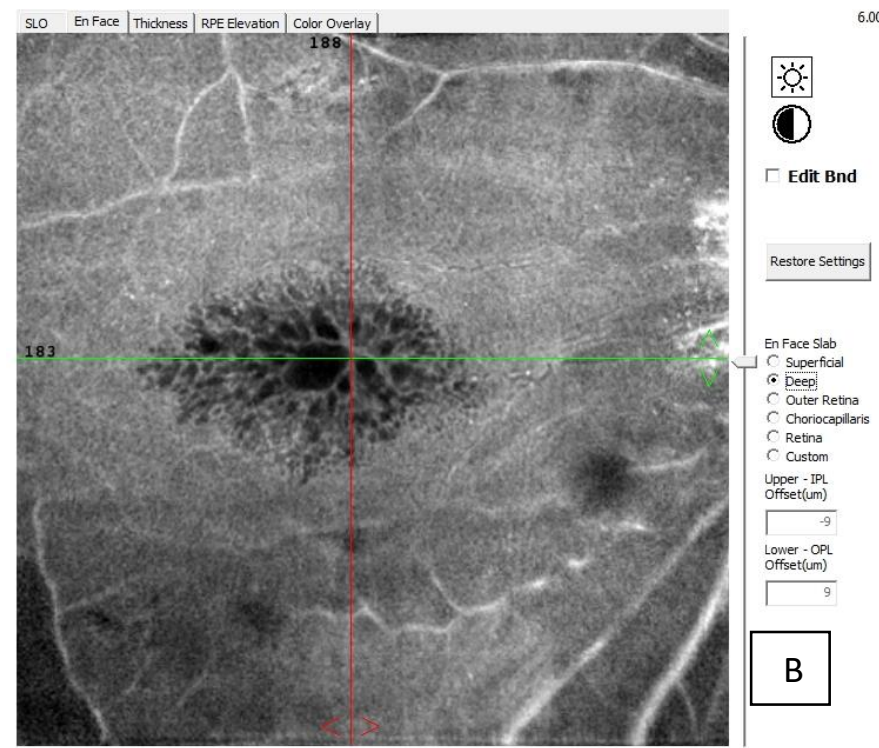

Figure 2: Right eye of a male patient 63 years old. En Face OCT image at level of superficial capillary plexus showing diffuse folding pattern with small cystic spaces(A), and at level of deep capillary plexus showing cavities of cystoid macular edema $(B)$.

\section{Star fold pattern of ERM}

:an Quality $7 / 10$

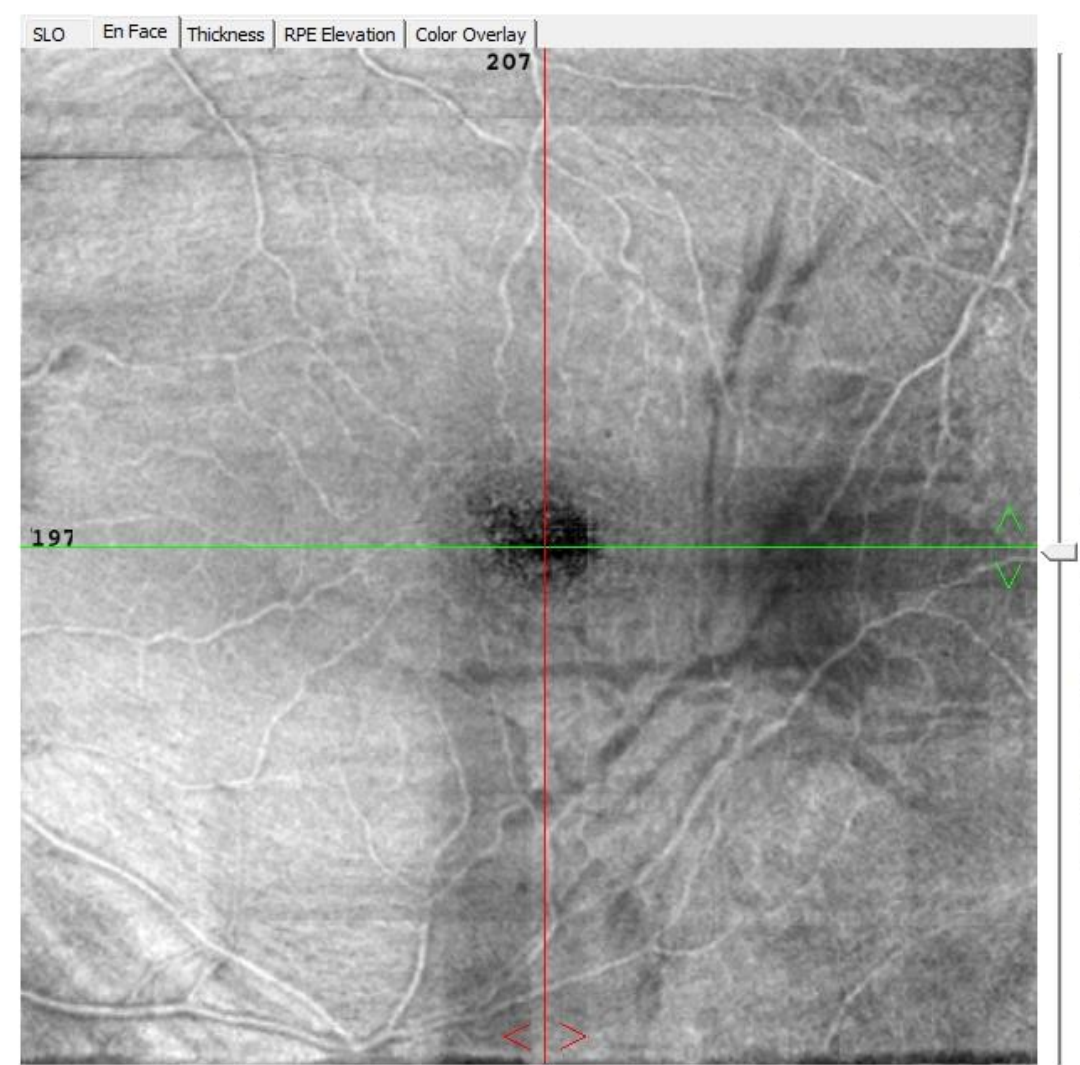

\section{Left / OS}

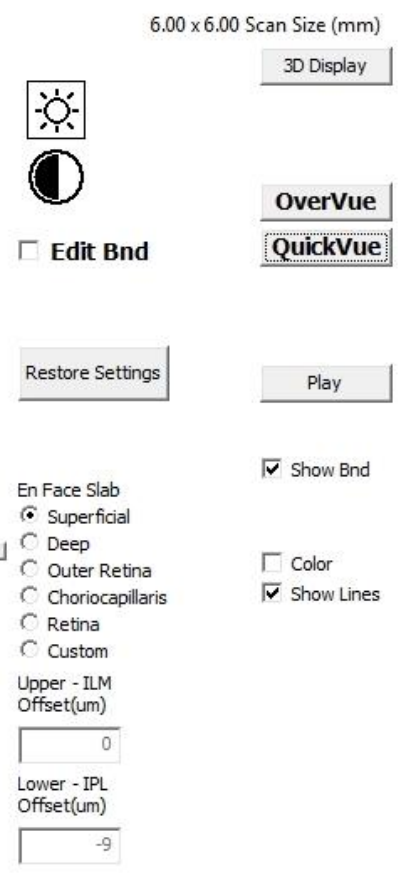

Figure 3: left eye of a male patient 75 years old. En Face OCT image at level of superficial capillary plexus showing a star fold pattern 
11. Diffuse folding pattern of ERM with retinal window

Figure 4: left eye of a male patient 61 years old. Enface OCT image at level of ILM showing a diffuse folding

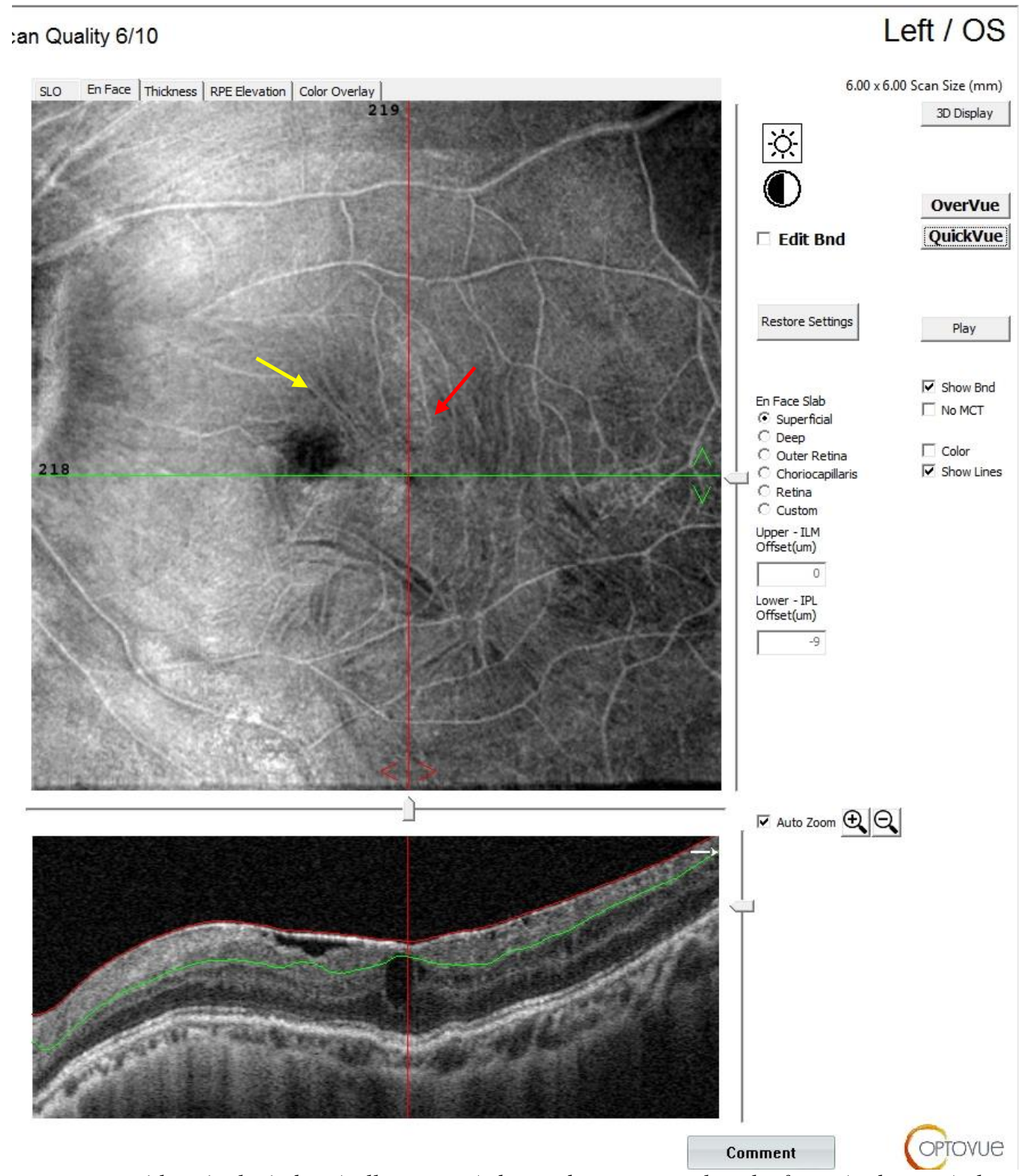

pattern with retinal window (yellow arrow) detected supero-nasal to the fovea (red arrow) showing the extent and location of ERM 


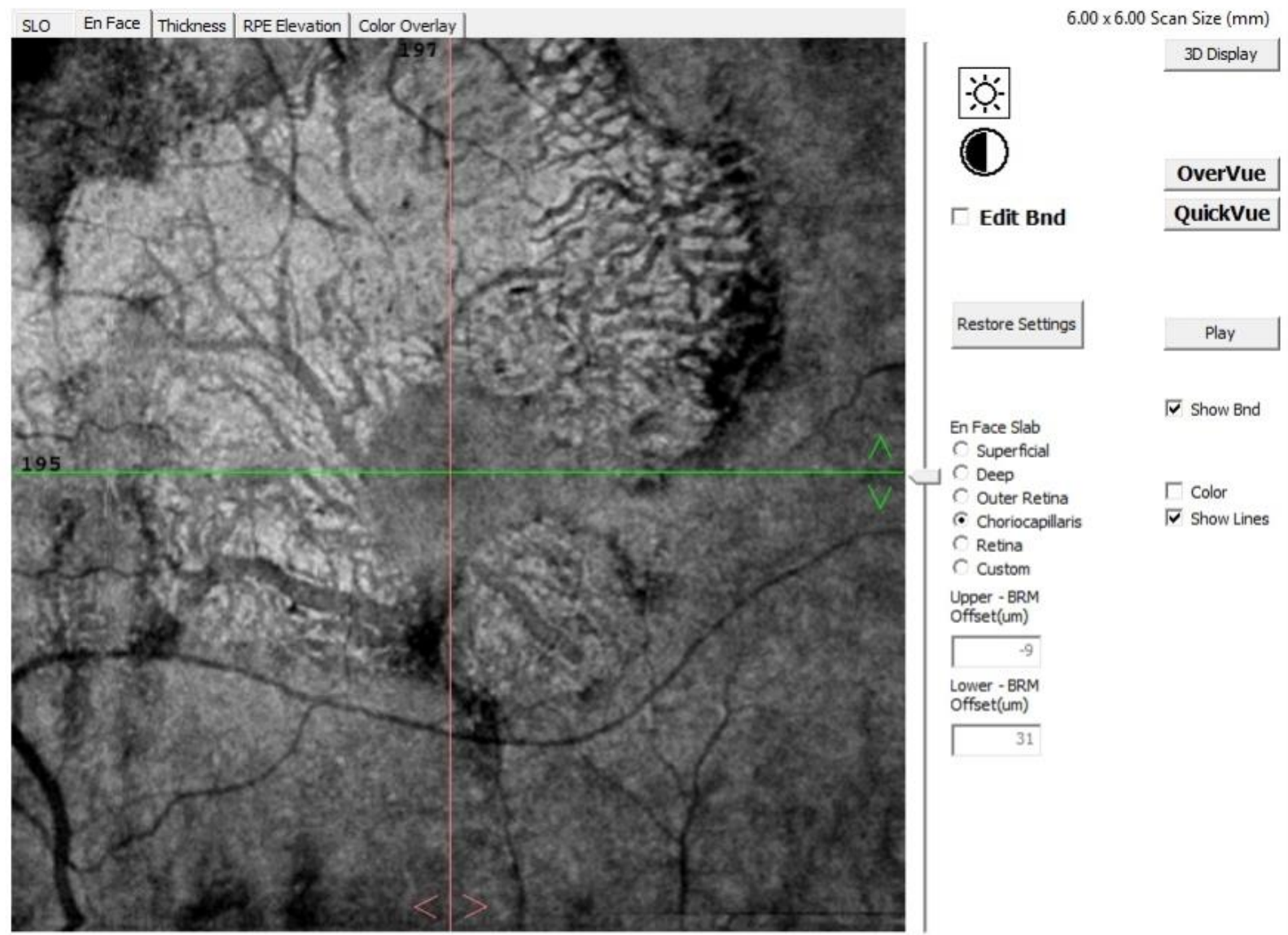

Figure 5: Left eye of male patient 83 years with geographic atrophy. enface OCT scan at level of choriocapillaris showing patches of loss of RPE and chorio-capillaris with visualization of large choroidal vessels denoting geographic atrophy.

13. Drusen with different sizes and drusenoid PEDS

Figure 6: Right eye of a female patient 52 years old. Enface OCT scan at level of outer retina (A) showing sub and para-foveal different sized whitish spots of drusen and drusenoid PEDs and at level of chorio-capillaris $(B)$ showing back shadowing of effect
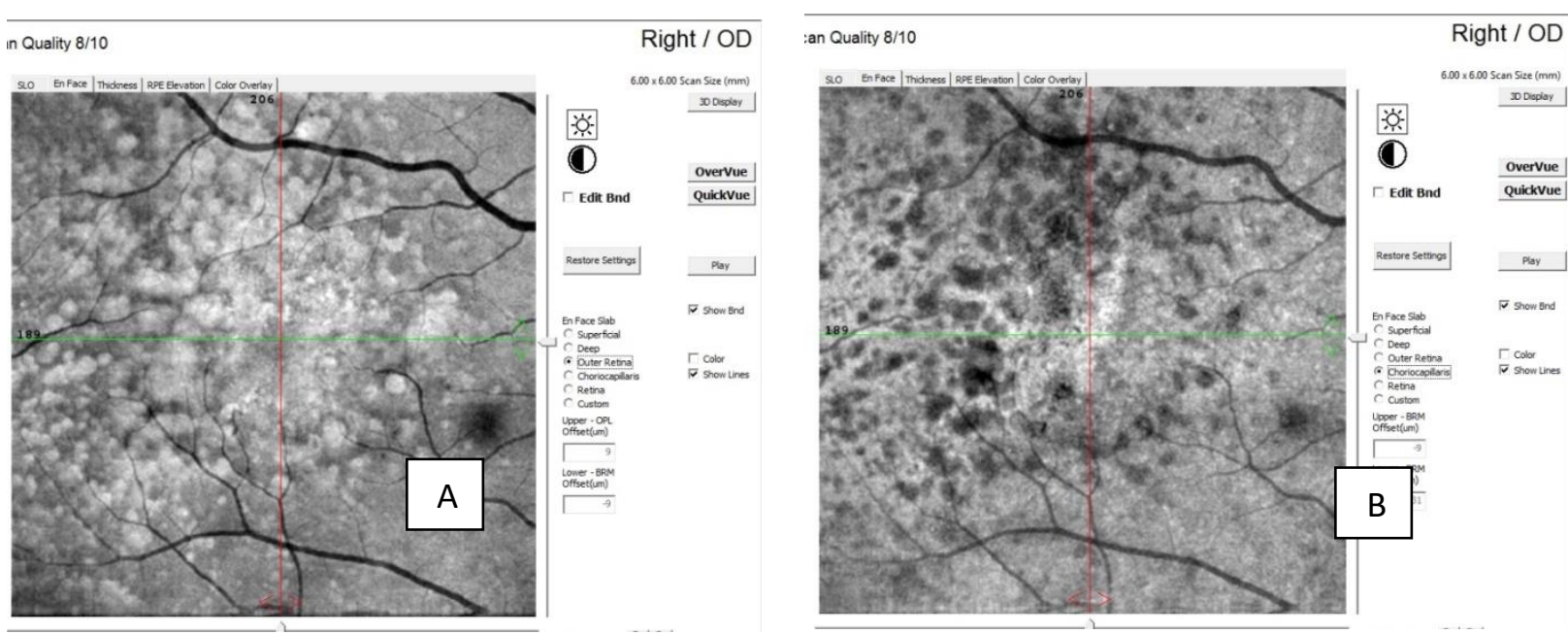
14. Active CNVM

Figure 7: right eye of male patient 65 years old. En face OCT image at the outer retina level showing CNVM

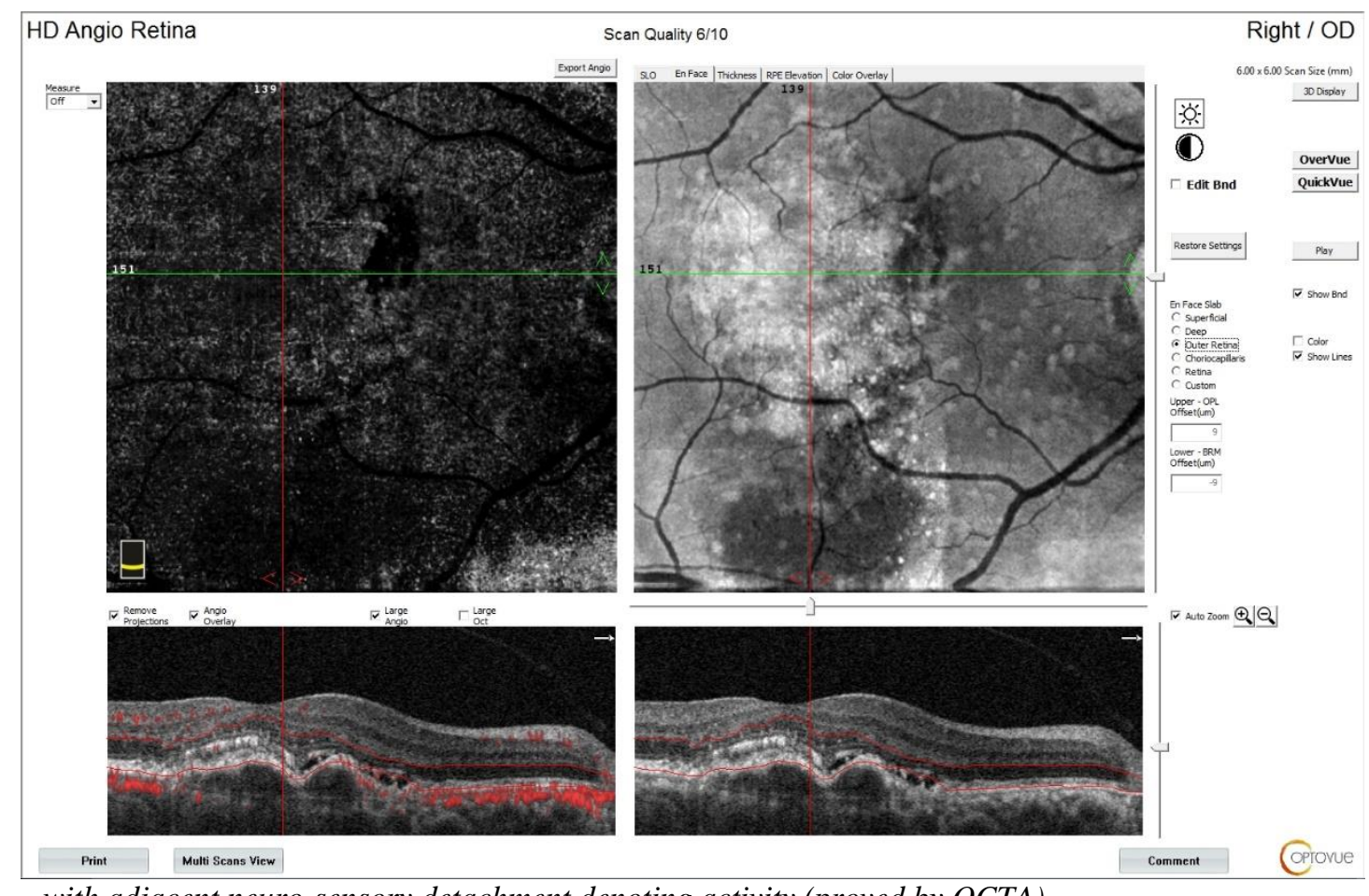

with adjacent neuro-sensory detachment denoting activity (proved by OCTA).

\section{Discussion}

The introduction of OCT technology has facilitated the examination of the macular area in a morphological way and in a quantitative way by measuring the macular thickness. En face OCT technology allows the frontal morphological examination in depth that is not available in B-scan OCT.

The question raised in this study was about the value of en face images in detecting different signs that help to diagnose macular pathology, do they have an added role in diagnosis?
The first author who described en face OCT as C-scan cuts of the posterior pole was Lumbroso and co-workers ${ }^{(6)}$. The highlighted specific findings in different lesions.

The added value of this study was to evaluate the role of en face OCT in detecting different signs of epi-retinal membrane and age related macular degeneration.

In this study, 30 eyes of 24 patients were examined to assess the role of en face 
OCT in diagnosis of some macular disorders.

Seventeen eyes of vitreo-macular interface disorders and 13 eyes of age related macular degeneration were diagnosed.

\section{1- Epi-retinal membrane (17 eyes) :}

In the examined cases of epi-retinal membrane, en face OCT detected epimacular plaques in 3 eyes, star folds in 2 eyes, diffuse macular folding in 10 eyes and mixed type in 1 eye. The fovea was affected with a shallow or lost foveal depression in 12 eyes and the fovea was not affected in 5 eyes. The value of en face OCT was that it detected the extent of the pathology over the macular area and areas of traction, although it could not detect a case with tightly adherent ERM with no traction.

It was demonstrated that spectral domain en face optical coherence tomography (SD-OCT) scanning of the retinal surface revealed plaques surrounded by radiating folds because of ERM. It discloses areas possibly devoid of internal limiting membrane (ILM) due to ERM contraction. In addition, it was mentioned that en face (SD-OCT) of the retinal surface is a good complement to retinal B-scan OCT that provides a wide overview of the retinal surface that is easy-to-understand. It also can identify progressive changes of the retinal surface occurring after internal limiting membrane (ILM) peeling ${ }^{(7)}$.

\section{2- Active CNVM (1 eye) and Fibro- vascular PED (1 eye):}

In our study, en face OCT detected one eye with fibro-vascular pigment epithelial detachment (FV-PED) and one eye of active CNVM, but did not confirm the presence of activity. When OCT angio was added, it confirmed that there is activity. This did not match the results of other study conducted ${ }^{(8)}$ where it was stated that "Until now, CNVs themselves were not precisely detectable in conventional Bscan OCT, but only suggested by the exudative reaction. En face OCT technology with dynamic segmentation of the macula allows detection of the contours and the shape of the FV-PED and the choroid neovascular network in the FV-PED. He also stated that en face SDOCT could demonstrate direct signs of choroidal neovascularization without any dye injection for the first time.

The miss-matching may happened due to the need for more levels of segmentation and limited number of cases.

\section{3- Geographic atrophy (2 eyes) :}

In this study, en face OCT detected geographic atrophy. Its value was that it could define the area of loss of photoreceptor, RPE and chorio-capillaris 
that is considered as a base line for monitoring the extent and progression of GA and the ability to measure the area of the pathology.

It was mentioned that en face OCT has also shown utility in conjunction with fundus auto-fluorescence (FAF) to predict progression of geographic atrophy (GA) in dry AMD ${ }^{(9)}$. While FAF shows hyperfluorescence at the border of advancing GA, en face OCT of the inner segment/outer segment junction demonstrated areas of darkening that corresponded to the progression over the next year in approximately one-third of patients.

\section{4- Drusen and derusenoid PED ( 9 eyes) :}

En face OCT was able to detect different lesions of dry AMD like small and large drusen and drusenoid PEDs. It is valuable in detecting the extent, type; size and number of lesions that may help in grading of the pathology, the need for anti-oxidant supplementation and as a base line for follow up.

It was previously mentioned (10) that drusenoid PEDs En face SD-OCT shows similar findings of soft drusen with a larger dimension of the RPE elevation. With this modality, the extension of the
RPE detachment is more clearly delineated than with other imaging techniques

It must be noted that limited studies about en Face OCT in all entities make it difficult to compare our study with other studies.

\section{Conclusion}

In conclusion, "en face" OCT is a novel technology offering a deeper insight into the understanding and follow up of macular disease.

When used alone, en face OCT can detect some useful information, but it is better to be used as an additive tool to B-scan OCT and OCT-angiography.

We recommend that more studies should be performed using this easy and useful technology, since the current research database lacks much information so far. More studies on each disorder can be performed separately with a larger number of patients and recording the observations for further correlation.

\section{References}

1. Wong I, Yong T, Lee J, Wong R, Iu L, Chhablani J et al. En-face optical coherence tomography in the diagnosis and management of age-related macular degeneration and polypoidal choroidal vasculopathy. Indian Journal of Ophthalmology. 2015;63(5):378. 
2. Zhou Q and Wei J. En face image formation and interpretation. In Lumbroso B, Huang D, Romano A, et al. (editors). Clinical En Face OCT Atlas. 1st ed. Jaypee Brothers Medical Publisher (P) Ltd.; 2013. P. 15-21.

3. Levison A and Kaiser P. Vitreomacular interface diseases: Diagnosis and management. Taiwan Journal of Ophthalmology. 2014; 4(2):63-68.

4. McKibbin M, Farragher T, and Shickle D. Vitreoretinal interface abnormalities in middle-aged adults with visual impairment in the UK Biobank study: prevalence, impact on visual acuity and associations. BMJ Open Ophthalmology. 2017;1(1):e000057.

5. Klein R, Rowland ML, and Harris MI. Racial/ethnic differences in age-related maculopathy: Third National Health and Nutrition Examination Survey. Ophthalmology. 1995;102(3):371-81.

6. Rispoli M, Le Rouic J, Lesnoni G, Colecchio L, Catalano S, and Lumbroso B. Retinal Surface En Face Optical Coherence Tomography. Retina. 2012;32(10):2070-2076.
7. Wang $\mathrm{M}$ and $\mathrm{Xu} \mathrm{G}$. Retinal venous occlusion. In Lumbroso B, Huang D, Andre R, Marco R, Gabriel C. (editors). Clinical En Face OCT Atlas. 1st ed. Jaypee Brothers Medical Publisher (P) Ltd.; 2013. P. 257-269.

8. Coscas F, Coscas G, Querques G, Massamba N, Querques L, Bandello F et al. En Face Enhanced Depth Imaging Optical Coherence Tomography of Fibrovascular Pigment Epithelium Detachment. Investigative Opthalmology \& Visual Science. 2012;53(7):4147.

9. Holz FG, Bellman C, Staudt S, Schütt F, Völcker HE. Fundus autofluorescence and development of geographic atrophy in agerelated macular degeneration. Invest Ophthalmol Vis Sci. 2001;42(5):1051-1056.

10. Eandi CM, Alovisi C, Tridico F, Grignolo FM. Spectral-domain optical coherence tomography and drusen: the en face modality. In Lumbroso B, Huang D, Andre R, Marco R, Gabriel C. (editors). Clinical En Face OCT Atlas. 1st ed. Jaypee Brothers Medical Publisher (P) Ltd.; 2013. P. 164-169.

To cite this article: Yasser N. Elkholy, Ayman H. Nassar, Zeinab S. Elsanabary, Ahmed E. Daifalla, Elham A. Gad. Evaluation of En-Face OCT Imaging in the diagnosis of macular diseases. BMFJ 2022;39: 90-107. (ophthalmology): DOI: 10.21608/bmfj.2021.97672.1489 
Benha medical journal vol. 39, special issue (ophthalmology), 2022 